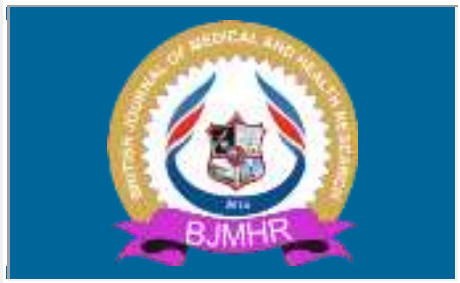

\title{
BJMHR
}

British Journal of Medical and Health Research

Journal home page: www.bjmhr.com

\section{Fumaria species - An Elaborative Pharmacological Review}

\author{
P.L.Rajagopal $^{{ }^{*} \text {, }}$ Rasila Jasmin $^{1}$, K.R.Sreejith ${ }^{1}$, K.Premaletha ${ }^{1}$, S.Aneeshia ${ }^{2}$ \\ 1.Department of Pharmacognosy and Phytochemistry, Academy of Pharmaceutical Sciences, \\ Pariyaram Medical College, Kannur, Kerala, India. \\ 2.Department of Medical Laboratory Technology, Academy of Paramedical Sciences, \\ Pariyaram Medical College, Kannur, Kerala.
}

\section{ABSTRACT}

Plant preparations are said to be medicinal or herbal when they are used to promote health beyond basic nutrition. It is indeed quite interesting to observe that our ancients were duly equipped with the vast, in depth and elaborated knowledge of drugs from the vegetable origin but unfortunately they possessed a scanty knowledge with regard to the presence of chemically pure compounds in most of them. In this review an attempt has been made to compile the reported pharmacological activities of the three Fumaria species. The aim of the present review is to find out the common pharmacological activities of the three Fumaria species and the review revealed that all the three are strong antioxidants and possess antimicrobial, anticancer, antiinflammtory, antiulcer activities. They are also used in treating skin diseases, as an hepato protectant and have the capacity to inhibit Acetylcholin esterase and butyrylcholin esterase activities.

Keywords: Fumaria indica, Fumaria parviflora and Fumaria vaillantii. 


\section{INTRODUCTION}

As per data available over three-quarters of the world population relies mainly on plants and plant extracts for their health care needs. More than $30 \%$ of the entire plant species, at one time or other was used for medicinal purposes. Treatment with medicinal plants is considered very safe as there is no or minimal side effects. These remedies are in sync with nature, which is the biggest advantage. The ancient scholars only believed that herbs are only solutions to cure a number of health related problems and diseases.

The synonym for Fumaria indica Linn. (Papavaraceae) are Fumaria parviflora Lamk. and Fumaria vallantii Loisel ${ }^{1}$. It has recently been identified sixty species of Fumaria and from those sixty species, Fumaria parviflora, Fumaria indica, and Fumaria vaillantii are closely associated species ${ }^{2}$. Even though, we have many references that Fumaria parviflora and Fumaria vallantii are the synonym for Fumaria indica, many researchers have mentioned their pharmacological activities separately and even references are there that these plants are designated as controversial plants.

\section{Fumaria parviflora}

\section{Anti-inflammatory activity}

Evaluation of anti-inflammatory activity of leaves of Fumaria parviflora were carried out on alcoholic and aqueous extract of the leaves of the plant. The anti-inflammatory activity was studied using carrageenan-induced paw edema method and cotton pellet granuloma method. The leaves of $F$. parviflora possess anti-inflammatory activity as they inhibit various cytokines and have antioxidant effects and free radical scavenging activity ${ }^{3}$.

In another study, 100 micro liters of carageenan 1\%was subcutaneously injected into the rat's paw and then the volume of the rat's paws were evaluated using plethysmometer apparatus. Once every hours between the first and fifth hours 200 and $400 \mathrm{mg} / \mathrm{kg}$ doses of extract had less effect on decreasing the paw's edema in comparison with animal group received aspirin ${ }^{4}$.

\section{Male fertility agent}

In the present study, healthy adult male rats were treated with $F$. parviflora leaves extract via gavage for 70 days. Blood samples were collected for determination of testosterone, LH and FSH serum levels. Significant increase was found in epididymal sperm density and percent of morphologically normal sperm in extract-treated rats. The results indicated that ethanolic extract of $F$. parviflora leaves have a potential to improve reproductive parameters and enhance male fertility ${ }^{5}$.

In another study, Fumaria parviflora was reported to be a medicinal plant with fertility indices promoting properties. Medicinal plants that are used in Iran affect hormonal compounds and other effective compounds on hormonal system as well as the proliferation 
and viability of sex cells due to their antioxidant properties, and therefore enhance fertility in human and animals ${ }^{6}$.

\section{Spermatogenesis}

The effect of Fumaria parviflora alcoholic extract on male rat spermatogenesis were evaluated. F parviflora was administered orally for 5 days through oral gavage. Experimental and control rats were sacrificed on day fifteenth after the first gavage. The testes were removed and analyzed. The weight and volume of the testes was increased in experimental groups but these increases were not significant. Moreover histopathological analysis showed that $F$. parviflora significantly increased the number of spermatogonium, spermatocytes, spermatozoids and Leydig cells. study indicate positive effect of $F$. parviflora alcoholic extract on male reproductive system because the number of sexual cells has increased significantly ${ }^{7}$.

Another study was conducted to investigate the effect of the $F$. parviflora alcoholic extract in spermatogenesis process. The control group received distilled water whereas the experimental group received $250 \mathrm{mg} / \mathrm{kg}$ w.b. F. parviflora alcoholic extract through oral gavage for 5 days. After 15 days rats were anesthesized and sacrificed and the testis were removed. The volume and weight of the testis were measured and the tissues were fixed. The analysis of the results indicated there were no significant difference in weight and volume of testicles but significant increase in the number of spermatogonia,spermatocytes, spermatozoid and leydig cells were observed which indicates positive effect of $F$. parviflora alcoholic extract on male reproductive system ${ }^{8}$.

\section{Testicular toxicity protective effect}

A study was carried out to investigate whether ethanolic extract of $F$. parviflora leaves could protect the male rats against lead-induced testicular oxidative stress. Adult Wistar rats were treated with and the lead acetate treatment resulted in significant reduction in testis weight, seminiferous tubules diameter, epididymal sperm count, and serum testosterone level, testicular content of superoxide dismutase and glutathione peroxidase. Moreover, significant elevation was observed in content of malondialdehyde in lead-treated rats. The results indicated that ethanolic extract of $F$. parviflora leaves prevented lead-induced testicular toxicity in male wistar rats 9 .

\section{Laxative and spasmodic effect}

Isolated intestinal preparations like ileum and jejunum from different animal species were separately suspended in tissue baths containing Tyrode's solution bubbled with carbogen and maintained at $37^{\circ} \mathrm{C}$. The spasmogenic responses were recorded using isotonic transducers 
coupled with Power Lab data acquisition system. This study shows the prokinetic, laxative and spasmodic effects of the plant ${ }^{10}$.

\section{Hepatotoxicity}

The effects of long term use of Fumaria parviflora on serum concentrations of total protein, glucose, cholesterol, triglyceride, aspartate aminotransferase, alanine aminotransferase and blood urea nitrogen were investigated. After 30 days of administration of $300 \mathrm{mg} \mathrm{kg}^{-1}$ of the extract caused a significantly higher serum concentration of aspartate amino transferase. According to the results of the present study long-term administration of $F$. parviflora extract with lower doses than that reported by other authors may cause hepatic damage ${ }^{11}$.

\section{Hepatoprotective activity}

The ethanolic extract of the aerial parts of Fumeria parviflora and Momordica balsamina were subjected to hepatoprotective assay by using wistar albino rats. Liver injury was introduced in rats by using carbon tetrachloride. The biochemical parameters like; serum glutamate, oxalo acetate transaminase, serum glutamate pyruvate transaminase, alkaline phosphatase and total bilirubin were estimated as a reflection of the liver condition. Based on these biochemical results a histopathological examination of the liver was carried out on the extract treated rats which shows normal appearance of hepatocytes which indicates the hepatoprotective effect of the plants. All results were comparable with silymarin ${ }^{12}$.

Another study was conducted to evaluate hepatoprotective activity of Fumaria parviflora leaf extract in isoniazid and rifampicin-induced hepatotoxic rats. It was found that pre-treatment of rats with $F$. parviflora leaf extract an hour prior to start isoniazid and rifampicin resulted in a significant decline in the serum levels of aspartate aminotransferase, alanine aminotransferase, alkaline phosphatase and total bilirubin as well as normal histology of liver biopsy specimens. It was concluded that $F$. parviflora leaf extract has significant hepatoprotective activity at the dose of $200 \mathrm{mg} / \mathrm{kg}$ comparable with that of silymarin. The plant extract appeared to have the potential to be used as a dietary supplement to antiTB therapy to protect against the hepatotoxicity of isoniazid and rifampicin ${ }^{13}$.

Nimesulide, a popular nonsteroidal anti-inflammatory drug, has been associated with serious hepatotoxicity. Study was undertaken to elucidate the effect of Fumaria parviflora Lam. on nimesulide induced cell death in primary rat hepatocyte cultures. The plant extracts treated cells showed increased viability as compared to nimesulide stressed cells as assessed by MTT assay. The study indicates that Fumaria purviflora extract modulates critical events regulating pro and anti-apoptotic proteins in mitochondria dependent apoptosis induced by nimesulide ${ }^{14}$. 


\section{Antidiabetic activity}

The present work was designed to evaluate the hypoglycemic effects of methanolic extract of $F$. parviflora in normal and streptozotocin-induced diabetic rats. Administration of $F$. parviflora extract showed a potent glucose lowering effect only on streptozotocin induced diabetic rats below $100 \mathrm{mg} / \mathrm{dl}$. The findings of the study indicated that $F$. parviflora has significant hypoglycemic effect on streptozotocin -induced diabetic rats with no effects on blood glucose levels of normal rats ${ }^{15}$.

In another study the blood glucose levels of the normal and alloxan-diabetic male albino rabbits were determined after oral administration of various doses of the powdered plants of Euphorbia prostrata, and Fumaria parviflora Lam. From the data obtained, it is concluded that the powders of $E$. prostrata and $F$. parviflora plants produce significant hypoglycemic effects in the normal rabbits only. In the alloxan-treated rabbits, these plants do not lower the blood glucose levels. It is conceivable that both plants contain some hypoglycemic principles which act probably by initiating the release of insulin from the pancreatic beta cells of normal rabbits ${ }^{16}$.

\section{Hypolipidemic activity}

Serum levels of glucose, triglyceride, total cholesterol, HDL and LDL were evaluated before and three and six weeks after the treatment with the plant extract. There was a significant decrease in triglyceride level in F. Parviflora L. treated group as compared to diabetic rats at third and sixth weeks. Oral administration of $F$. Parviflora L. to streptozocin-induced diabetic rats improved triglyceride, total cholesterol and HDL serum levels, but no significant effect on serum glucose and $\mathrm{LDL}^{17}$.

\section{Social aggression behaviour}

The effect of aqueous extract of the Fumeria parviflora was studied on social aggression behaviour of laboratory male MFI albino mice was studied using video camera along with some other plants. Aqueous extracts were given by intraperitoneally for fourteen days .Twenty minutes after last dose social aggression encountered was videotaped for ten minutes for each animal. The plant extracts produced a significant reduction in at least one of the elements in aggressiveness monitored. The study also revealed that the plants are hepatotoxic, neurotoxic and cardiotoxic. The plant is also shown CNS stimulant effects ${ }^{18}$.

\section{Anthelmintic activity}

The anthelmintic activity of Fumaria parviflora was evaluated against the gastrointestinal nematodes of sheep through egg hatch and larval development tests in vitro and faecal egg counts reduction test in vivo. The study showed that $F$. parviflora whole plant extracts possess anthelmintic activity, thus justifying their use in traditional veterinary practices ${ }^{19}$. 
In another study, eighty sheep naturally infected with fasciolosis were randomly divided in to four groups. Fumaria parviflora and Caesalpinia crista showed 59.4\%, 91.8\% and 58.7\%, $87.3 \%$ efficacy at the dose of $80 \mathrm{mg} / \mathrm{kg}$ on 18 and 28 day post treatment, respectively. It was concluded that Fumaria parviflora and Caesalpinia crista were comparable with that of standard drug oxyclozanide possessing significant anthelmintic activity against ovine fasciolosis ${ }^{20}$.

\section{Antinociceptive effects}

In this study the antinoiciceptive and histopathological effects of the methanolic extract of fumaria parviflora Lam. in animal models was investigated. The results showed that the percolated extract had significant antinociceptive effect in hot-plate. The study of ulcerogenic effects of oral percolated extract on stomach in rats showed that this adverse effect was significantly lower in comparison with the same dose of indomethacin. These results showed F. parviflora is relatively safe for use and it is a good candidate for further studies to determine its effective and probable toxic compound ${ }^{21}$.

\section{Uremic pruritic activity}

The study aimed to assess the efficacy of Fumaria parviflora for reducing uremic pruritus severity among hemodialysis patients. A total of 79 hemodialysis patients with pruritus were randomly assigned to receive either Fumaria parviflora or a placebo for eight weeks. Fumaria parviflora can significantly decrease the severity of uremic pruritus in hemodialysis patients $^{22}$.

\section{Nematicidal Activity}

Two bioactive nematicidal phytochemicals, viz., nonacosane-10-ol and 23a-homostigmast-5en-3 $\beta$-ol, were isolated from the $n$-hexane fraction of the roots of Fumaria parviflora through activity-guided isolation. Activity of the two compounds against eggs and juveniles (J2s) of Meloidogyne incognita was evaluated in vitro at the concentrations of 50, 100, 150, and 200 $\mu \mathrm{g} \mathrm{mL}{ }^{-1}$. Over $120 \mathrm{~h}$ of incubation, the cumulative percent mortality and hatch inhibition of both of the compounds tested ranged from 20 to $100 \%$ and from 15 to $95.0 \%$, respectively. These compounds could provide new insight in the search for novel nematicides against $M$. incognita $^{23}$.

\section{Acetylcholinesterase and butyrylcholinesterase inhibitory activity}

Fumaria parviflora Lam. and Fumaria judaica Boiss were screened for their anticholinesterase activity on acetylcholinesterase (AChE) and butyrylcholinesterase (BChE) enzymes by in vitro Ellman method at $10 \mathrm{microg} / \mathrm{ml}$ and $1 \mathrm{mg} / \mathrm{ml}$ concentrations. Fumaria extracts displayed highly potent inhibition against both of the enzymes at $1 \mathrm{mg} / \mathrm{ml}$ concentration compared to the standard ${ }^{24}$. 


\section{Antidiarrheal, antispasmodic and bronchodilator activities}

The aqueous-methanol extract of Fumaria parviflora protected against diarrhoea caused by castor oil in rats and mice, similar to loperamide and dicyclomine, and bronchospasm caused by carbachol $(\mathrm{CCh})$ in rats, similar to aminophylline. This study, showing the presence of antidiarrheal, antispasmodic and bronchodilator activities in Fumaria parviflora possibly mediated through dual blockade of muscarinic receptors and $\mathrm{Ca}(2+)$ channels, provides sound basis for its medicinal uses in diarrhea, abdominal cramps and may be used as bronchodilator in asthma ${ }^{25}$.

\section{In treating eczema}

This study aimed to evaluate the effect of alcoholic extract of Fumaria parviflora on hand eczema. In a randomized double-blind, placebo-controlled study, 44 patients with hand eczema were randomly assigned to apply $4 \%$ cream of Fumaria parviflora or vehicle cream to hand twice daily for 4 weeks. The reduction of eczema area and severity index score before and two weeks after therapy was statistically significant between vehicles treated and in treated group. Only one patient showed side effects such as erythema and population. Fumaria parviflora appears to be tolerated by most patients and the findings showed that its extract may be considered as an effective agent for treatment of chronic hand eczema ${ }^{26}$.

\section{Skin diseases}

The Fumaria and Licorice plants were extracted using maceration method. After concentration of extract and freeze-dried them, weight from every 100gram extract of Glycyrrhiza were $16.6 \mathrm{~g}$ and for Fumaria were $13.8 \mathrm{gr}$. The $\mathrm{pH}$ of formulation after 3 month was in the range of 5.7-6.7. The prepared formulation was stable in the experimental condition and this formulation can be selected for the clinical trial on skin disorders ${ }^{27}$.

\section{Anti protozoal activity}

Antiprotozoal effect of the ethanol extracts obtained from five Fumaria species like; Fumaria densiflora, Fumaria cilicica, Fumaria rostellata, Fumaria kralikii, and Fumaria parviflora was investigated against the parasites; Plasmodium falciparum (malaria) and Trypanosoma bruceirhodesiense The study shows its promising antiprotozoal activity ${ }^{28}$.

\section{Fumaria indica}

\section{Anti-inflammatory activity}

Oral administration of $F$. indica dry extract exhibited dose dependent and significant antiinflammatory activity in acute carrageenean and histamine induced hind paw oedema, and chronic cotton pellet granuloma models of inflammation ${ }^{29}$.

Anti-inflammatory activities of Fumaria indica (FI) and Fumaric acid (FA) were observed in carrageenan induced edema and cotton pellet granuloma even after their lowest tested doses. 
Significant analgesic activities of both FI and FA in hot plate and tale flick tests were observed after all their tested doses ${ }^{38}$.

Another study was conducted to evaluate the analgesic activity of the plant were, acetic acid induced writhing test Aspirin as $300 \mathrm{mg} / \mathrm{kg}$ orally was used as a reference compound. The crude extract of $F$. indica showed significant dose related inhibition of number of writhes ${ }^{39}$.

\section{Cognitive function modulating effects}

$50 \%$ ethanolic extract of $F$. indica $L$. was evaluated for putative cognitive function modulating effects. Suspension of the plant in $0.3 \%$ carboxy methyl cellulose was orally administered to rats during the entire experimental period of 16 days at dose levels of 100 , 200, and $400 \mathrm{mg} / \mathrm{kg} / \mathrm{day}$. The plant showed dose-dependent decrease in brain AChE activity and increase in muscarinic receptor density. The plant possesses nootropic-like beneficial effects on cognitive functions ${ }^{30}$.

\section{Antisecretory, gastroprotective and antacid capacity}

Evaluation of $F$. indica extract as antisecretory was carried out by pyloric ligation induced ulcer model. The gastroprotective effect was carried out by absolute ethanol induced ulcer model. Integrity of gastric mucosa was evaluated by estimation of GSH and gastric mucous level. The in-vitro antacid capacity was evaluated -1by titration method. Ethanol extract of $F$. indica at $200 \mathrm{mg} \mathrm{kg}$, orally showed inhibition of secretion in pyloric -1 -1 ligation model. GSH level, gastric wall mucous and percentage protection of ulcer were significantly increased in absolute ethanol induced ulcer model ${ }^{31}$.

\section{Antimicrobial Activities}

In vitro antimicrobial activities of various concentrations ranging from 100 to $500 \mathrm{mg} / \mathrm{ml}$ of alcoholic (methanol) extracts of Fumaria indica were analyzed on different clinical bacterial strains and fungal strains using agar disk diffusion method and broth dilution method for antimicrobial activity. In vitro antimicrobial activity results revealed that methanol extract of $F$. indica possess greater antibacterial activity than antifungal activity ${ }^{32}$.

In an another study, the antibacterial activity of the crude methanolic extract and various fractions of $F$. indica were determined against Escherichia coli, Pseudomonas aeruginosa, Staphylococcus aureus and Bacillus subtilus. Zone of inhibition was measured (in $\mathrm{mm}$ ) in comparison with positive control. The results show potent antibacterial activity to the plants $^{33}$.

In an another study, solvent extracts of Mazus japonicus and Fumaria indica were investigated individually and in combinations for their antibacterial potency against Bacillus subtilis, Pseudomonas geniculata, Streptomyces laurentii, Klebsiella pneumoniae, 
Microbacterium oxydans, Alcaligenes faecalis, and Enterococcus faecium. Minimum inhibition concentration and fractional inhibitory concentration were investigated ${ }^{34}$.

Another study investigated antibacterial potential of aerial parts of Fumaria indica and Silybum marianum against planktonic and biofilm form of 3 clinical strains P1, P2 and P3 of Pseudomonas aeruginosa. Antibacterial activity against planktonic form was investigated by Well diffusion method. Crude methanolic extracts of Fumaria indica showed good antibacterial activity against $\mathrm{P}$. aeruginosa with maximum $15.8 \mathrm{~mm}$ zone of inhibition (ZOI) against P2 strain of P. aeruginosa and minimum $13.2 \mathrm{~mm}$ zone of inhibition against P3 $\operatorname{strain}^{35}$.

\section{Antioxidant activity}

An in vitro evaluation model utilizing a $\beta$-carotene bleaching assay and DPPH free radical scavenging test was employed for the assessment of antioxidant potential of the plant extract. A modified Folin-Ciocalteu method used for total phenolic. The findings of this research adjudged the extract of $F$. indica a rich source of natural antioxidant and endowed with antimicrobial activity ${ }^{36}$.

\section{CNS depressant activity}

Antidepressant activity of the extract was also evaluated using validated models of depression in rodents viz. Behavioural despair test, learned helplessness test, tail suspension test, reserpine induced hypothermia, 5-Hydroxytryptophan (5-HTP) induced head twitches in mice and L-dopa induced hyperactivity and aggressive behaviour in mice. It may be concluded that the plant has significant central nervous system depressant activity and lacking antidepressant activity in rodents ${ }^{37}$.

\section{Phytotoxic Activity}

The phytotoxic activity of $F$. indica test samples was measured against Lemna minor $\mathrm{L}$. The method of McLaughlin et al., (1991) was followed for this activity. Stock solutions of the test samples were prepared in methanol at concentration of $20 \mathrm{mg} / \mathrm{ml}$. E-medium was also prepared for the growth of L. minor. Results were taken by counting the number of damaged plantlets. Results show low phytotoxic activity ${ }^{33}$.

\section{Haemagglutination Activity}

The method of Naqvi et al., (1992) was followed. Fresh blood was collected from healthy volunteers on the day of experiment. Positive and negative results are indicated by rough granules and smooth button formation, whereas extent of deposition determined the intensity of positive result. The result shows low haemagglutination activity ${ }^{33}$. 


\section{Adaptogenic effect}

The present pilot study was designed to experimentally verify the possibility that fumarates are the major bioactive principles of Fumaria indica extracts involved in their stress response modulating activities, and to estimate pharmacologically active dose ranges of fumarates and standardized methanolic extract of Fumaria indica (MFI). Obtained results reveal high efficacy of MFI and pure fumarates possess qualitatively analogous activity profiles in all the three tests ${ }^{40}$.

\section{Nutrition effects}

The nutritional constituents of the plants were determined using spectroscopic, calorimetric, flame photometric, chemical assay methods. Two lots of each plant in duplicate were evaluated for the parameters protein, fiber, fat, carbohydrate, vitamin A \& $\mathrm{C}$, energy value, calcium, phosphorus etc Fumaria indica was found to contain protein content 10.68 -14.81 $\%$, fiber $23.59-28.97 \%$, carbohydrate $4.93-6.24 \%$ calcium $1.61-1.86 \%$, vitamin A 916.52- 1666.05 IU/g \& energy 300.25 - $356.75 \mathrm{Kcal} / 100 \mathrm{~g}$ Further study on livestock as feed material is needed to prove their nutritional efficacies ${ }^{41}$.

\section{Anticonvulsant Activity}

The present study aimed to investigate the role of several biologically active compounds from Fumaria Soy.-Will. in anticonvulsant models. According to empirical results, the expressed anticonvulsant effect of Fumaria dry extract depends on the synergism of biologically active compounds in herbal medicine, although some individual substances have shown moderate anti-seizure activity ${ }^{42}$.

\section{Antiulcer activity}

The present study was undertaken to evaluate the effects of roots of Daucus carota and aerial parts of Fumaria indica on gastric tissue glutathione level in experimental rats with indomethacin-induced gastric ulceration. Ethanol extract from roots of D. carota and aerial parts of $F$. indica was administered at the doses 100 and $200 \mathrm{mg} / \mathrm{kg} / \mathrm{day}$, p.o. Ulcer index, gastric GSH level and gastric wall mucous level were estimated From this study, it was concluded that ethanol extracts each from roots of D. carota and aerial parts of $F$. indica posses significant ulcer protection by replenishing Gastric GSH and increasing gastric mucous level ${ }^{43}$.

\section{Wound Healing activity}

Dried powdered whole plant of Fumaria indica was extracted with 50\% ethanolic extract. The extract was subjected to its wound contraction, pro-inflammatory and anti-inflammatory potentials. The $50 \%$ ethanolic extract showed presence of ellagic acid, ferulic acid and quercetin. The IC50 was $0.11 \mathrm{mg} / \mathrm{mL}$ and significant antibacterial activity was observed 
against S. aureus and E. coli. The $10 \%$ F. indica ointment applied topically to the wound area reduced its size from $500 \mathrm{~mm} 2$ to $40 \mathrm{~mm} 2$ by the end of 9th day. These results were comparable to the effect of $0.2 \%$ nitrofurazone. The extract further showed a reduction in the release of pro-inflammatory cytokines and an increase in anti-inflammatory cytokine ${ }^{44}$.

\section{Diuretic activity}

The diuretic activity of extracts of Fumaria indica was examined by treating different groups of wistar albino rats with single oral doses of alcoholic and aqueous extract/fractions. Furosemide was used as positive control in the study. Out of the different fractions and extract, the ethanolic fraction significantly increased the urine output and urinary electrolyte The results of this study strongly indicate the ethanolic fraction of Fumaria indica showed the greater diuretic effect ${ }^{45}$.

\section{Haemoprotective activity}

Phenyl hydrazine is used as a model for the induction of haemolytic anaemia in Charles foster rats. Flowers of Fumaria indica showed the remarkable haemo protective activity against the haemolytic anaemia. Methanolic extract of Fumaria indica showed the haemoprotective activity against phenyl hydrazine induced haemolytic anaemia in Charles foster rats at different doses. The plant extract contains this haemoprotective activity due to the presence of high phenolic, flavonoid and alkaloid contents ${ }^{46}$.

\section{Hepatoprotective activity}

The present investigation demonstrates the hepatoprotective potential of $50 \%$ ethanolic water extract of whole plant of Fumaria indica and its three fractions viz., hexane, chloroform and butanol against d-galactosamine induced hepatotoxicity in rats. The hepatoprotection was assessed in terms reduction in histological damage, changes in serum enzymes (SGOT, SGPT, ALP) and metabolites bilirubin, reduced glutathione (GSH) and lipid peroxidation (MDA content) ${ }^{47}$.

\section{Acne vulgaris}

In the present study aqueous extract of Fumaria indica were selected for analyses, efficacy and safety in the treatment of acne vulgaris. The study design was randomized, single blind, placebo controlled containing thirty (30) cases including both sexes which were divided into two groups. Group 1 received test drug (Fumaria indica) and group 2 received placebo orally, for a period of 6 weeks. Patients followed for three visits after every two weeks. Results were extremely significant in group 1 , and moderately significant in group 2 , These findings together with prior preclinical knowledge of the plant, suggest that Fumaria indica could be an easily accessible source for discovering and developing phyto pharmaceuticals or drugs potentially useful for acne vulgaris ${ }^{48}$. 


\section{Fumaria vaillantii}

\section{Anticancer activity}

The aim of the present study was to determine the anti-proliferative potential of Fumaria vaillantii extracts on three different cancer cell lines including malignant melanoma SKMEL3, human breast adenocarcinoma MCF-7 and human myelogenous leukemia K562 as well as human gingival fibroblast (HGF) as normal cell line. Anti-proliferative activity was evaluated by 3-(4,5-dimethylthiazol-2-yl)-2,5-diphenyltetrazolium bromide (MTT), flowcytometry and annexin methods. Total phenolics and flavonoids were determined by Folin-Ciocalteu and aluminum chloride methods. The collective data demonstrated that $F$. vaillantii chloroform fraction may contain effective compounds with chemo-therapeutic potential act through an apoptotic independent pathway ${ }^{49,50}$.

\section{Hepatoprotective activity}

In the present study, effect of monomethyl fumarate and Fumaria extract was investigated against acetaminophen-induced acute liver damage and compared to a known hepatoprotective plant, Silybum marianum and its active ingredient, silymarin. Biochemical parameters including AST, ALT, LDH, ALKP and total and direct bilirubin were measured in serum samples. Glutathione and Malon Di Aldehyde contents were determined in mouse liver homogenates. Liver sections were also collected for histopathological examination. The results indicate that monomethyl fumarate and Fumaria extract exhibited hepatoprotective action against acetaminophen liver injury which is comparable to that of Silybum marianum and silymarin ${ }^{51}$.

In an another study, the hepatoprotective activity of ethanol extracts of Fumaria vaillantii Lois. was investigated against $\mathrm{CCl}_{4}$-induced toxicity in rats. F. vaillantii $(500 \mathrm{mg} / \mathrm{kg})$ caused a significant decrease in the plasma aspartate aminotransferase (AST), alanine aminotransaminase (ALT) and tissue malondialdehyde levels, and a significant increase in the reduced glutathione and catalase levels, compared with the $\mathrm{CCl}_{4}$ group. These findings were approved by histopathological observations. Additionally, a significant decrease was found in liver $\mathrm{Cu}$ levels $(18.5 \%)$ of the extract-treated group $(500 \mathrm{mg} / \mathrm{kg})$, while an increase in liver $\mathrm{Zn}(16.2 \%)$ levels was observed compared with the $\mathrm{CCl}_{4}$ group. These changes in liver $\mathrm{Zn}$ and $\mathrm{Cu}$ levels caused by $F$. vaillantii extract may contribute to its positive effects on liver toxicity ${ }^{52}$.

\section{Anti-inflammatory activity}

Oral administration of the ethanolic extract (200 and $400 \mathrm{mg} / \mathrm{kg}, \mathrm{p} . \mathrm{o}$ ) of the aerial parts of Fumaria vaillantii Loisel produced significant analgesic activity in acetic acid-induced 
writhing and tail immersion tests, anti- inflammatory effect against carrageenan induced inflammation and adjuvant induced polyarthritis ${ }^{53}$.

\section{Antiulcer activity}

The plant extract significantly attenuated gastric mucosal damage induced by toxic agents and indomethacin in rats. Aqueous extract of the plant restored partially mucous secretion in indomethacin model. In pylorus-ligated rats, aqueous extract significantly reduced the basal gastric acid secretion, acidity and ulceration. Aqueous extract possesses significant and more potent anti-ulcer and cytoprotective activities against experimentally-induced gastric ulcers in comparison to Omeprazole ${ }^{54}$.

\section{In the management of hypochlorhydria}

Hypochlorhydria was induced by ranitidine in this study. Hypochlorhydria causes iron deficiency in rat and it was prevented significantly by the extract treatment at the ratio of $1: 1$ of the said plant and fruit. The correction of iron deficiency by the composite extract was compared with iron supplementation to hypochlorhydric rat. It was found that pre administration followed by co administration of FVBH-1 (1:1) able to prevent the ranitidineinduced hypochlorhydria and iron deficiency. The composite extract, FVBH-1 (1:1) significantly $(P<0.05)$ increased the pepsin concentration, chloride level in gastric juice, iron levels in serum and liver along with blood hemoglobin level than other ratios used here. Hence, it can be concluded that FVBH-1 (1:1) is an effective herbal formulation for the management of hypochlorhydria and related iron deficiency ${ }^{55}$.

\section{Acetylcholinesterase and butyrylcholinesterase inhibitory activity}

The extract of the plant was screened for their anticholinesterase activity on acetylcholinesterase (AChE) and butyrylcholinesterase (BChE) enzymes by in vitro Ellman method at $10 \mu \mathrm{g} / \mathrm{ml}$ and $1 \mathrm{mg} / \mathrm{ml}$ concentrations. The extract of the plant did not show any noticeable inhibitory activity against both of the enzymes at $10 \mu \mathrm{g} / \mathrm{ml}$. Fumaria extracts displayed highly potent inhibition against both of the enzymes at $1 \mathrm{mg} / \mathrm{ml}$ concentration compared to the standard ${ }^{56}$.

\section{Antimicrobial and antioxidant activity}

Different parts of the plant Fumaria vaillantii was extracted with three different solvents to yield 72 crude extracts. The phytochemical analysis (chemical screening, GC-MS) of three plants was investigated for their antioxidant and antibacterial activity using nine Grampositive and Gram-negative bacteria. The principal antioxidant and antimicrobial components were determined using HPLC with UV detection. All extracts possessed antibacterial activity ${ }^{57}$. 


\section{Hypochlorhydric activity}

In this study hypochlorhydria was $(\mathrm{pH} \geq 4)$ induced by ranitidine, a histamine receptor antagonist, and the aim of this present study is to evaluate the protective effect of preadministration followed by co-administration of aqueous extract of whole plant of Fumaria vaillantii L. (AEFV) or ripe fruit of Benincasa hispida T. (AEBH) in individual as well as by composite manner in ranitidine-induced hypochlorhydric rat. We measured the $\mathrm{pH}$, free acidity and total acidity, vitamin $\mathrm{C}$ concentration of gastric juice along with levels of vitamin $\mathrm{C}$ in plasma and hemoglobin in blood. These herbal extracts recovered the gastric secretion and it gave satisfactory results in vitamin $\mathrm{C}$ concentration in gastric juice though the composite extract is more potent than individual in this aspect. This study highlighted the protective activity of these two plants from the hypochlorhydric state ${ }^{58}$.

\section{Antifungal activity}

The main oil content from the plant of $F$. vaillantii was $0.25 \%(\mathrm{v} / \mathrm{w})$ and that essential oil was analyzed by capillary gas chromatography (GC) using flame ionization (FID) and capillary gas chromatography coupled mass spectrometry (GC/MS) for detection. Eighteen compounds were identified in the essential oil of $F$. vaillantii that included $99.62 \%$ of the total oil. The major components were Parfumidine (18.94\%), Fumaricine (16.30\%), Thymol (12.45\%) and Fumaritine (10.78\%). The study of the antifungal effects of the oil sample was carried against strain of Aspergillus flavus (PTCC=5004) by disc diffusion method via average inhibition zone. The results show that the essential oil from fumaria plant at 1 and $1 / 2$ oil dilutions exhibited strong antifungal activity than gentamycin antibiotic on A. flavus and synthetic thymol exhibited good inhibition at $10 \%$ dilution. Large percentage antifungal activities of fumaria oil are related with thymol as a natural monoterpene phenol is the main compound ${ }^{59}$.

\section{CONCLUSION}

All the three are strong antioxidants and possess antimicrobial, anticancer, anti-inflammatory, antiulcer activities. They are also used in treating skin diseases, as an hepato protectant and have the capacity to inhibit Acetylcholine esterase and butyrylcholine esterase activities. Few researchers are commented that these three are closely related species and few are commented about the synonym factor. Since most of the researchers are considering these three are different species of Fumaria a detailed study on the taxonomical characters of these three species are necessary for the confirmation.

\section{REFERENCES}

1. Gireesh K. Singh, Sudhir K. Chauhan, Geeta Rai,Shyam S. Chatterjee, and Vikas Kumar. Potential antianxiety activity of Fumaria indica: A preclinical study. Pharmacognosy Magazine.2013;9(33):14-22. 
2. Sharma PV. Dravyaguna-Vijnana, Chaukhambha Orientalia Varanasi: 2 nd ed., 2003;320-22.

3. Waseem Rizvi1, Mohammad Fayazuddin, Ompal Singh, Syed Shariq Naeem, Shagufta Moin, Kafil Akhtar, Anil Kumar. Anti-inflammatory effect of Fumaria parviflora leaves based on TNF- $\alpha$, IL-1, IL-6 and antioxidant potential. AJP, 2017;7(1):37-45.

4. Arzi, Ardeshir and Nazari, Zahra and Ahmadi Salianeh, Seied Vahid .Effect of Fumaria Parviflora hydro alcoholic extract on induced carageenan inflammation in male rat paw. Jentashapir Journal of Health Research,2013; 4 (2): 121-130.

5. Mehran Dorostghoal, Seyyed Mansoor Seyyednejad, Lotfollah Khajehpour and Ayoob Jabari. Effects of Fumaria parviflora leaves extract on reproductive parameters in adult male rats. Iran J Reprod Med.2013;11(11):891-898.

6. Mahnaz Yavangi. Use of Iranian Medicinal Plants Effective on Male Fertility Indices. Journal of global pharma technology.2016;8(10).

7. Mitra Heydari Nasrabadi, Helia Aboutalebi and Maryam Naseri. Effect of Fumaria parviflora alcoholic extract on male rat's reproductive system. Journal of Medicinal Plants Research. 2012;. 6(10):2004-2010.

8. Maryam Naseri, Mitra HeydariNasrabadi, Parvin Khodarahmi, Faribosadat Ahmadi, Parisa Mojibi and Helia Abotalebei. Study of the Effect of Fumaria parviflora Alcoholic Extract on Spermatogenesis in Male Rats. New cellular and molecular biotechnology journal.2011;1(2):61-65.

9. Dorostghoal M, Seyyednejad S.M and Jabari A. Protective effects of Fumaria parviflora L. on lead-induced testicular toxicity in male rats. Andrologia.2014;46(4):437-446.

10. Najeeb-ur-Rehman, Malik Hassan Mehmood, Adnan J Al-Rehaily, Ramzi AA Mothana, and Anwar H Gilani. Species and tissue-specificity of prokinetic, laxative and spasmodic effects of Fumaria parviflora. BMC Complementary and Alternative Medicine 2012;12(16):1-8.

11. Javad Tajik, Saeed Nazifi and Fahimeh Poorzal. The Effects of Long-term Use of Fumaria parviflora extract on Some Serum Biochemical Parameters of Rats. Journal of Pharmacology and Toxicology.2011; 6:710-714.

12. Saleh I, Alqasoumi, Mohammad S and Al-Dosari.Evaluation of hepatoprotective effect of Fumeria parviflora and Momordica balsammina from Saudi folk medicine against experimentally induced liver injury in rats. Research journal of medicinal plant. 2009;3(1):9-15. 
13. Khan H M and Iqbal S .Comparative Hepatoprotective Activity of Fumaria farviflora Lam. Leaf Extract and Silymarin on Isoniazid and Rifampicin-induced Hepatotoxic Rats. Indian Journal of Pharmaceutical Sciences.2017;79(1):124-130.

14. M Tripathi, BK Singh, C Mishra, S Raisuddin, $\mathrm{P}$ Kakkar. Involvement of mitochondria mediated pathways in hepatoprotection conferred by Fumaria parviflora Lam. extract against nimesulide induced apoptosis in vitro. Toxicology in vitro.2010; $24(2), 495-508$.

15. Fatemeh Fathiazad, Sanaz Hamedeyazdan, Mohamad Karim Khosropanah and Arash Khaki. Hypoglycemic Activity of Fumaria parviflora in Streptozotocin Induced Diabetic Rats. Advanced Pharmaceutical Bulletin.2013;3(1):207-210.

16. Akhtar MS, Khan QM and Khaliq T. Effects of Euphorbia prostrata and Fumaria parviflora in Normoglycaemic and Alloxan-treated Hyperglycaemic Rabbits.Planta Med 1984; 50(2): 138-142

17. Mohammad Hassan, Ghosian-Moghadam, Mehrdad Roghani, Zahra Mohammadnezhad and Arezoo Mehrabian. Fumaria parviflora Lam. Effect on Serum Levels of Glucose and Lipids in Streptozocin- Induced Diabetic Rats. Journal of basic and clinical pathophysiology.2014;2(2):35-42.

18. Mansour A AL-Hazmi.Effect of some medicinal plant extract on aggressive behaviour of laboratory mice. JKAU: Sci. 2002;14:13-23.

19. Al-shaibani IRM, , Phulan MS and Shiekh M. Anthelmintic Activity of Fumaria parviflora (Fumariaceae) against Gastrointestinal Nematodes of Sheep. Int. J. Agric. Biol.2009; 11(4):431-436

20. Anisa Mushtaq, Nisar Ahmad, Haroon Akbar, Aneela Zameer Durrani, Muhammad Waqas, Mohsin Nawaz, Zulfiqar Ahmad, Sajid Mahmood Sajid, Muhammad Akhtar Qureshi and Abid Hussain. Therapeutic Efficacies of Fumaria parviflora, Caesalpinia crista and Oxyclozanide against Fasciolosis in Naturally Infected Sheep in Rawalakot-Azad Jammu and Kashmir, Pakistan. World Journal of Zoology.2015; 10 (2): 118-123.

21. Mahmood reza heidari, Ali mandgary, Mohsen enayati . Antinociceptive effects and toxicity of Fumaria parviflora lam. in mice and rats. DARU.2004;12(4):136-140.

22. Rahimeh Akrami, Mohammad Hashem Hashempur, Ali Tavakoli, Majid Nimrouzi, Mehrab Sayadi, Maryam Roodaki, Jamshid Roozbeh and Pouya Faridi. Effects of Fumaria parviflora L on Uremic Pruritus in Hemodialysis Patients:A randomized double blind placebo controlled trial. Juindishapur jounal of Natural Pharmaceutical Products.2016;1-6. 
23. Ishrat Naz, Saifullah and M. R. Khan. Nematicidal Activity of Nonacosane-10-ol and 23a-Homostigmast-5-en-3 $\beta$-ol Isolated from the Roots of Fumaria parviflora (Fumariaceae). J. Agric. Food Chem., 2013; 61 (24):5689-5695.

24. Orhan I, Sener B, Choudhary MI, and Khalid A. Acetylcholinesterase and butyrylcholinesterase inhibitory activity of some Turkish medicinal plants. J Ethnopharmacol. 2004; 91(1):57-60.

25. Najeeb-ur-Rehman, Bashir S, Al-Rehaily AJ and Gilani AH. Mechanisms underlying the antidiarrheal, antispasmodic and bronchodilator activities of Fumaria parviflora and involvement of tissue and species specificity. $\mathrm{J}$ Ethnopharmacol. 2012;144(1):128-37.

26. Jowkar F, Jamshidzadeh A, Mirzadeh Yazdi A, Pasalar M. The effects of fumaria parviflora L extract on chronic hand eczema: a randomized double-blind placebo controlled clinical trial._Iran Red Crescent Med J. 2011;13(11):824-828.

27. Siahpush, Amir; Moravej, Maryam Sadaat; Makhmalzadeh and Behzad Sharif. Formulation and Evaluation of an Herbal Cream from Dry Extract of Glycyrrhiza glabra and Fumaria parviflora. Medical Journal of Tabriz University of Medical Sciences \& Health Services .2014; 36 ( 5):40-47.

28. Ilkay Erdogan Orhan, Nilgun Ozturk, Bilge Sener. Antiprotozoal assessment and phenolic acid profiling of five Fumaria species. Asian Pacific journal of tropical medicine2015;8(4):283-286.

29. Chandana venkateswara rao, Arti raj verma , Praksh kumar gupta and Madhavan vijayakumar. Anti-inflammatory and anti-nociceptive activities of Fumaria indica whole plant extract in experimental animals. Acta Pharm. 2007;57: 491-498.

30. Gireesh Kumar Singh, Geeta Rai, Shyam Sunder Chatterjee and Vikas Kumar. Effects of ethanolic extract of Fumaria indica L. on rat cognitive dysfunctions.AYU. 2013;34(4):421-429.

31. Phool Chandra, Kamal Kishore and Ashoke Kumar Ghosh . Evaluation of antisecretory, gastroprotective and in-vitro antacid capacity of Fumaria indica in rats. Journal of Environmental Biology.2015; 36:1137-1142.

32. Abida Khan, Hidayatullah Tak, Ruqiaya Nazir, Bashir A Lone and Javaid A Parray. In Vitro Anthelmintic and Antimicrobial Activities of Methanolic Extracts of Fumaria Indica. Clinical Microbiology. 2014;3(5):1-5.

33. Ibrar khan, Haroon Ahmad, Nasir Ali, Bashir Ahmad, Sadiq Azam and Fida Hassan. Screening of Fumaria indica for antibacterial, antifungal, phytotoxic and 
haemagglutination activities. International journal of current biotechnology.2013;1(9):13-16.

34. Naila Safdar, Nilofar Yaqeen, Zehra Kazmi and Azra Yasmin. Antimicrobial Potential of Mazus japonicus and Fumaria indica Extracts: Individual vs. Synergistic Effect Journal of herbs, spices and medicinal plants.2017;23(4).

35. Imran Khan, Azhar Hussain Shah, Wajiha Khan and Uzma Khan. In vitro antibacterial activity of Fumaria indica and Silybum marianum L. against planktonic and biofilm form of Pseudomonas aeruginosa. International Journal of Biosciences.2016;8(4):94-102.

36. Ajay Kumar Sharma , Prakash Chandra Gupta and Rao CHV. Total Phenolic Content, Antioxidant and Antimicrobial Activity of Fumaria indica (Hausskn.) Pugsley. DDD. 2014; 1 (2) : 103 - 109.

37. SinghG. K and Kumar V. Neuropharmacological screening and lack of antidepressant activity of standardized extract of Fumaria indica: a preclinical study. Electronic Journal of Pharmacology and Therapy.2010;3: 19-28.

38. Anshul Shakya, Gireesh Kumar Singh, Shyam Sunder Chatterjee and Vikas Kumar. Role of fumaric acid in anti-inflammatory and analgesic activities of a Fumaria indica extracts. J Intercult Ethnopharmacol. 2014; 3(4): 173-178.

39. http://www.pspuok.com/books/monograph\%20on/25.pdf

40. Anshul Shakya, Shyam Sunder Chatterjee and Vikas Kumar. Role of fumarates in adaptogenics like efficacies of traditionally used Fumaria indica extracts.TANG.2015;5(1):1-10.

41. Kotagiri Ravikanth, Anil Kanaujia, Deepak Thakur, Anirudh Sharma and Bhupesh Gauta. Nutritional Constituents of the Plants Fumaria indica and Caesalpinia bonducella. IJAPBC .2014; 3(3):698-702.

42. Yuliya Prokopenko, Vadim Tsyvunin, Sergey Shtrygol and Victoriya Georgiyants. In Vivo Anticonvulsant Activity of Extracts and Protopine from the Fumaria schleicheri Herb Sci. Pharm. 2016;84, 547-554.

43. Chandra P., Kishore K., Ghosh A. K. Effect of Daucus carota and Fumaria indica on gastric tissue glutathione level in indomethacin induced ulcers. Indian Drugs, 2015;52(5):12-16.

44. Pandey Garima, Kumar G Rajesh, Gupta Shyam Sundar, Ojha S K, Rao Ch V. Wound Repair and Anti-inflammatory Potential of Fumaria indica in Excision Wound-induced Rats. British Journal of Pharmaceutical Research. 2014;4(2):257-266. 
45. Ramesh Kumar Gupta, Sudhansu Ranjan Swain, Jagannath Sahoo, Padala Narasimha Murthy, Pramod Kumar Sharma and Umakant Bajaj Comparative study of diuretic potential of aqueous and alcoholic extracts of Fumaria indica in experimental rodents. European Journal of Experimental Biology, 2016; 6(1):4-8.

46. Raj Kumar and Vivek Kumar Mishra. Phenylhydrazine induced haematotoxicity and it's recovery by Fumaria indica plant extract in charles foster rats. Anil Division of Toxicology ,CSIR-Central Drug Research Institute,Lucknow-226031, Uttar Pradesh, India, International Journal of Scientific and Innovative Research 2016; 4(2) : 22-31

47. A Rathi, AK Srivastava, A Shirwaikar, AKS Rawat Hepatoprotective potential of Fumaria indica Pugsley whole plant extracts, fractions and an isolated alkaloid protopine. Phytomedicine, 2008;15(6-7):470-477.

48. Masarat Fatima1, Asia Sultana2, Mohd. Asif 3, Firdous Ahmad Najar. Efficacy and safety of Fumaria indica (aqueous extract) in the treatment of acne vulgaris International Journal of Institutional Pharmacy and Life Sciences.2014;4(6): 47-58.

49. Fatemeh Haji Abbas Tabrizi Saeed Irian,Amir Amanzadeh, Fatemeh Heidarnejad, Hoda Gudarzi, and Mona Salimi. Anti-proliferative activity of Fumaria vaillantii extracts on different cancer cell lines. Res Pharm Sci. 2016;11(2): 152-159.

50. Fatemeh Haji Abbas Tabrizi, Saeed Irian, Amir Amanzadeh, Fatemeh Heidarnejad, Hoda Gudarzi, and Mona Salimi. Anti-proliferative activity of Fumaria vaillantii extracts on different cancer cell lines Research in Pharmaceutical Sciences, 2016; 11(2): 152-159.

51. Ezzat Zamani-Moghaddam, Kian Azami, BagherMinaei-Zangi, Seyedeh Zahra Mousavi and Omid Sabzevari. Protective effect of Fumaria vaillantii extract and monomethyl fumarate on acetaminophen induced hepatotoxicity in mice.2012; $8(3): 177-184$.

52. Didem Deliorman, Ali Hartevioglu, Nilufer Orhan, Aysel Berkkan, Alper Gokbulut, Omer Gunhan and Mer Pekcan Subacute. Effects of Standardized Fumaria Vaillantii Lois. Ethanol Extract on Trace Element Levels, Biochemical and Histopathological Parameters in Experimental Liver Toxicity.2016;40(2):180-189.

53. Pankaj nainwal, Kapil kalra, Deepak nanda and Amandeep singh. Study of analgesic and anti-inflammatory activities of the ethanolic extract arial parts of Fumaria vaillantii loisel. Asian Journal of Pharmaceutical and Clinical Research. 2011;4(1):90-91. 
54. Rifat-uz-Zaman and Attiq-ur-Rehman. Anti-helicobacter pylori and protective effects of aqueous Fumaria vaillantii L extract in pylorus ligated, indomethacin- and toxicinduced ulcers in rats. Afr.J.Pharm.Pharmacol.2010;4(5):256-262.

55. Upanandan Mandal, Kazi Monjur Ali, Kausik Chatterjee, Debasis De, Anjan Biswas, Debidas Ghosh. Management of experimental hypochlorhydria with iron deficiency by the composite extract of Fumaria vaillantii L. and Benincasa hispida T. in rat. Journal of Natural Science, Biology and Medicine.2014;5(2):397-403.

56. OrhanBŞener, M.IChoudhary and AKhalid. Acetyl cholinesterase and butyryl cholinesterase inhibitory activity of some Turkish medicinal plants. Journal of EthnopharmacologyVolume.2004;91(1):57.

57. Jaberian, Hamideh, Piri, Khosro and Nazari, Javad. Phytochemical composition and in vitro antimicrobial and antioxidant activities of some medicinal plants. Food chemistry, 2013;136: 237-244

58. Mandal U, Nandi D, Chatterjee K, Biswas A, Ghosh D. Remedial effect of aqueous extract of whole plant of Fumaria vaillantii Loisel and ripe fruit of Benincasa hispida Thunb in ranitidine induced-hypochlorhydric male rat. International Journal of Applied Research in Natural Products.2010; 3 (1):37-47.

59. Moghtader M. In vitro antifungal effects of Fumaria vaillantii Loisel. essential oil on Aspergillus flavus. Journal of yeast and fungi research 2013;4(2): 21-25.

60. Suresh Kumar, Anil Kumar Sharma and Anjoo Kamboj . Fumaria parviflora Lam. (Fumitory): A traditional herbal medicine with modern evidence.Asian Journal of Pharmacy and Pharmacology 2017; 3(6): 200-207.

61. Mansoor Ahmad, Mehjabeen, Mahboob Alam, Noor Jahan, Farah Saeed and Zahid Khan. Neuropharmacological and biological activities of Fumaria indica. International Journal of Phytomedicine.2015;7:62-68.

62. Sweta Srivastava and G.P Choudhary. Pharmacognostic and pharmacological study of Fumaria vaillantii Loisel: a review. Journal of Pharmacognosy and Phytochemistry 2014; 3 (1): 194-197.

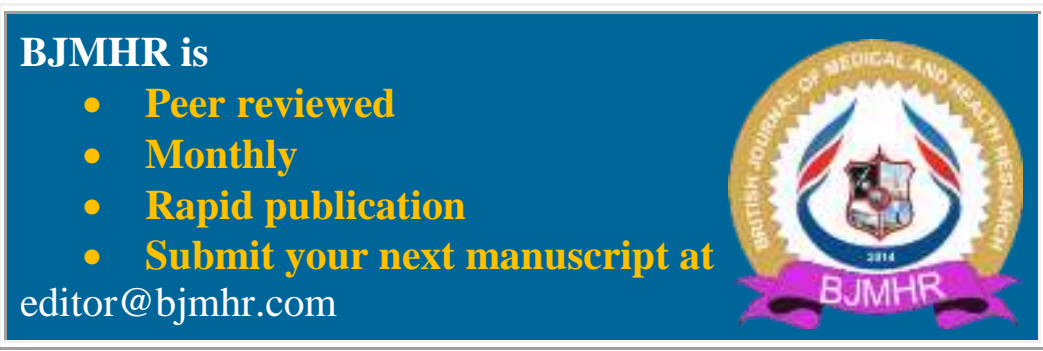

